\title{
Reduction of ocular muscle power by splitting of the rectus muscle I: Biomechanics
}

\author{
T Haslwanter, R Hoerantner, S Priglinger
}

Br J Ophthalmol 2004;88:1403-1408. doi: 10.1136/bjo.2004.042713

Background/aim: Based on mechanical considerations, the authors have developed a new approach to weakening oculomotor muscles. They present the biomechanical considerations that have encouraged them to explore this approach, and compare it with existing surgical techniques. Results of application to patients are given in the companion paper, and do not require an analytical understanding of the underlying mechanics.

Methods: Using a simple biomechanical model for the oculomotor system and vector component analysis, the eye position dependent torque exerted by extraocular muscles on the eyeball was investigated. This model was applied to the healthy eye, as well as to different surgical procedures (Cüppers' Fadenoperation, Y-split muscle recessions, botulinum toxin, and simple muscle recessions).

Conclusion: These investigations suggest that a Y-split muscle recession is a simple and efficient way to weaken ocular rectus muscles. Compared to alternative surgical procedures, undesired radial forces that can lead to post-surgical complications can be kept to a minimum. The authors further speculate that their good results may in part be because of possible preservation of proprioceptive inputs from the insertion of the extraocular muscle.
W hile many strabismological problems can be resolved by non-invasive procedures, some require surgical intervention to weaken one or more extraocular muscles. Depending on the type of muscle, different surgical procedures can be used for that purpose: unilateral or bilateral muscle recessions, with or without muscle resection $^{1-4}$; "Cüppers' Fadenoperation," also referred to as "posterior fixation" or "retroequatorial myopexy" ${ }^{\prime 5-15}$; or treatment of the overactive muscle with botulinum toxin. ${ }^{16-18}$ All these techniques affect the underlying mechanics of the oculomotor plant differently, and thus have different effects on the oculomotor system, as well as different side effects. Theoretical calculations, which are based on a drastically simplified model of the oculomotor system ${ }^{19}$ and which will be outlined below, suggest that a Y-splitting combined with recession of a rectus muscle reduces the torque generated by that muscle. ${ }^{19} 20$ This torque reduction is caused by a shortening of the effective lever arm of the muscle.

An exact representation of the action of each of these methods would require complex, detailed models. Nevertheless, it is possible to investigate the principles of the underlying mechanics through a relatively simple analysis of the underlying geometry, based on a mechanical model in which the extraocular muscles are represented by simple strings. While our model does not explicitly include muscle pulleys, we demonstrate that the existence of these structures should not change the underlying mechanisms for weakening the rectus muscles.

\section{METHODS}

To a first approximation, the movement of the human eye can be regarded as a pure rotation of the eyeball in its socket. Therefore, the relevant entity to consider is not the force $F$ exerted by each extraocular muscle onto the eyeball, but the torque $T$ exerted by each muscle, with the lever arm $r$ :

$$
T=F * r
$$

To demonstrate this, consider two children, one big, the other very slim, sitting on a seesaw (fig 1A): as expected, the heavy child will easily push its side down. In our comparison, this corresponds to one muscle in a muscle pair exerting too big a torque (fig 1B).

To get the seesaw balanced, we have a number of different options (fig 2):

(a) We can starve the big child until it loses weight. This could be compared to a muscle recession in which the muscle gets weakened by shortening the muscle path from its origin to its insertion on the globe.

(b) As we will show below, the Cüppers surgery does not decrease the strength of the rectus muscle, but changes its pulling direction such that only part of the muscle force contributes to the torque rotating the eye. The remaining force acts in a radial direction on the eyeball. In our comparison, only part of the weight of the big child contributes to the torque on the seesaw, while the other part compresses the spring. ${ }^{5} 6101221$

(c) The effect of the bigger child can also be reduced by shortening the lever arm of the seesaw. This corresponds to the shortening of the lever arm of the muscle in the Y-split recession operation, as demonstrated below. ${ }^{19}$

\section{Mathematical description}

An accurate description of the forces exerted by the extraocular muscles onto the eyeball is a highly complex entertainment. It has to include the mechanical structures attaching the extraocular muscles (EOMs) to the orbita, also referred to as "pulleys", as well as their innervation, ${ }^{22}$ the length-tension relation of the EOMs, the retrobulbar fatty tissue, muscle-muscle interaction (for example, "Lockwood's ligament"), and the correct geometry of these structures. In these models-for example, Orbit by Miller $^{23}$ or SEE++ by Buchberger and coworkers, ${ }^{24}{ }^{25}$ the forces generated by the EOMs are derived in an iterative way and cannot be expressed analytically-that is, with closed formulas. To

Abbreviations: EOMs, extraocular muscles 
A

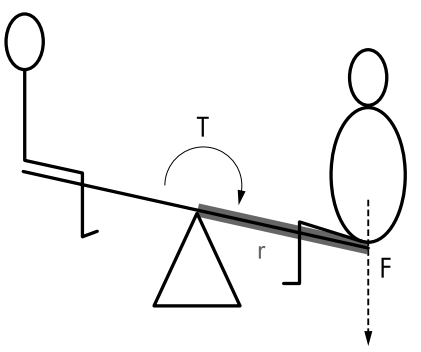

B

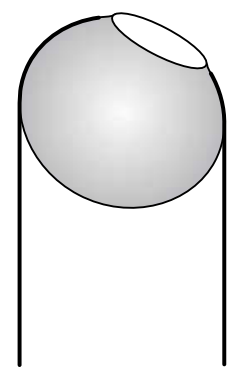

Figure 1 (A) Children on a seesaw: the heavier child pushes the seesaw down, since the torque $T$ generated by the weight $F$ and the lever arm $r$ is bigger than the torque generated by the lighter child. (B) Strabismic eye: the overactive muscle pulls the eye away from the desired orientation, inducing the observed strabismus.

explain the underlying mechanics of the different treatment approaches mentioned above, we therefore resort to rough simplifications. This will allow us to obtain simple formulas, which may help to understand the mechanisms through which different operations affect the forces exerted by the EOMs onto the eyeball.

We will describe the EOMs as infinitely long strings, which attach at a small point onto the eyeball, and whose path is affected neither by other EOMs nor by muscle pulleys. In the discussion we will explain the effects of these assumptions on the results of our model.

\section{Healthy eye}

To introduce the mathematical description of the forces and torques exerted by one muscle on the eye, it is helpful to start out with the consideration of a healthy subject.

In figure 3 , the eye of the subject has a radius $r_{\text {eye }}$. When the subject looks straight ahead, the length of the muscle path along the globe is denoted with $\mathrm{l}_{\mathrm{mg}}$ (fig $3 \mathrm{~A}$ ). The rotational movement of the eye will be denoted by $\delta$. For small eye movements-that is, eye movements with $\delta \leqslant \Phi_{\max }$ with (fig 3A)

$$
\Phi_{\max }=l_{\mathrm{mg}} / r_{\text {eye }}
$$

the full force $\mathrm{F}$ contributes to the torque $\mathrm{T}$ exerted by the muscle on the eye, which is given by

$$
T=F * r_{\text {eye }}
$$

Values for a typical patient are $\mathrm{r}_{\text {eye }}=11 \mathrm{~mm}$ and $\mathrm{l}_{\mathrm{mg}}$ $=5.8 \mathrm{~mm} \rightarrow \Phi_{\max }=30^{\circ}$. But when the movement gets larger-that is, $\delta>\Phi_{\max }$, only part of the force can contribute to the rotating torque. Figure $3 \mathrm{C}$ shows that for such large angles, the force $\mathrm{F}$ acting on the eye can be decomposed into two components: one component $\left(\mathrm{F}_{\text {tang }}\right)$ acts in a tangential direction, thereby generating the torque exerted by the muscle on the eyeball

$$
\begin{aligned}
& F_{\text {tang }}=F * \cos (\alpha) \\
& \text { where } \\
& \alpha=\delta-\Phi_{\max }
\end{aligned}
$$

The resulting torque is

$$
T=F_{\text {tang }} * r_{\text {eye }}
$$

A

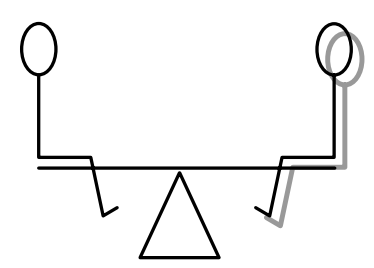

B

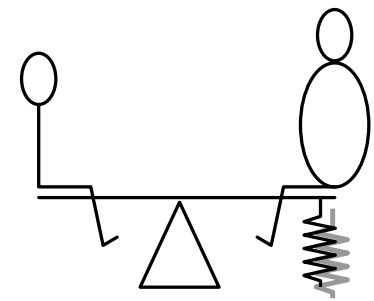

$\mathrm{C}$

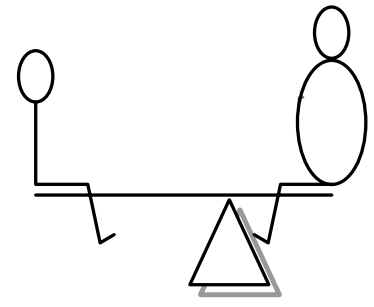

Figure 2 To balance the seesaw there are a number of options, which are indicated by shadows: (A) Make the heavy child lighter $(\rightarrow$ muscle recession). (B) Redirect some of the force away from the seesaw $(\rightarrow$ Cüppers' Fadenoperation). (C) Shorten the lever arm ( $\rightarrow$ Y-split recession).

Since $\cos (\alpha) \leqslant 1$ and the cosine function decreases with increasing angle, this implies that the rotating torque stays constant until $\delta$ reaches $\Phi_{\max }$, and then gets smaller (with the cosine function) as the rotation increases. The other component,

$$
F_{\text {rad }}=F * \sin (\alpha)
$$

acts in a radial direction. In other words, it pulls away from the eye, without contributing to the rotating torque. (In fig $2 \mathrm{~B}$, this would correspond to the force $F_{\text {rad }}$ being absorbed by the spring.)

\section{Cüppers' Fadenoperation}

In Cüppers' Fadenoperation, the rectus muscle is sutured to the globe. While it has been pointed out that a distinction between an orbital and a global layer of the rectus muscle allows a slightly different interpretation of the operation, ${ }^{26}$
A

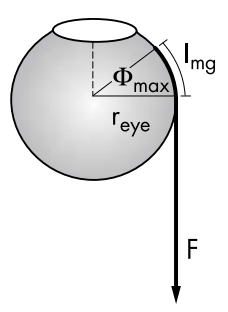

B

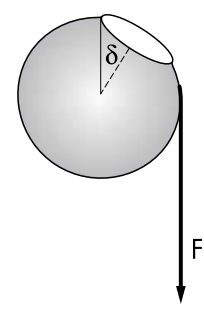

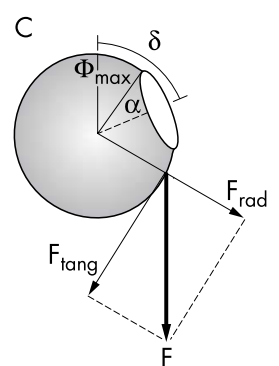

$\mathrm{C}$
Figure 3 Healthy eye: $(\mathrm{A}, \mathrm{B})$ For eye movements with $\delta<\Phi$ max the full force is used to generate a torque that rotates the eye. (C) For $\delta>\Phi_{\text {max, }}$ only part of the force applied to the muscle rotates the eye $\left(F_{\text {tang }}\right)$. The other part pulls in a radial direction, contributing nothing to the rotation $\left(F_{\text {rad }}\right)$. 
A
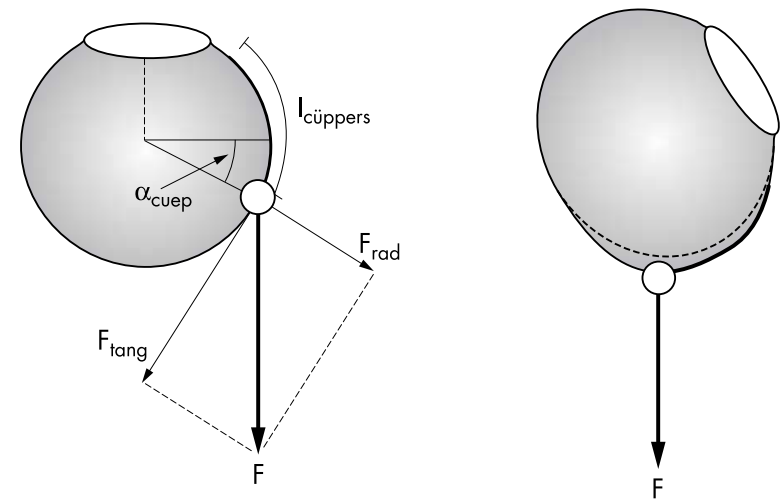

Figure 4 Cüppers' Fadenoperation. (A) Already in primary position, only the part $F_{\text {tang }}$ of the force $F$ applied to the eye contributes to the rotation of the eye. (B) For larger excursions, the eye can become effectively blocked.

we will for the moment side-step this point and concentrate on the central geometry underlying this surgery. In this operation the point of attachment of the EOM is shifted from the original insertion point to the location of the suture. The location of the suture mainly depends on the near and far squint angle, as well as on the diameter of the globe, and is commonly about $13-20 \mathrm{~mm}$ behind the natural insertion point of the muscle. If an effect on the static squint angle is necessary, the affected muscle can also be recessed. ${ }^{10-12} 142728$

Applying the algorithms developed above to that situation, we can see why this operation decreases the rotating torque of the eye: the effect of the Fadenoperation is to move the attachment point backwards, which means that $l_{m g}$ in equation (2) has to be replaced by $l_{m g}-l_{\text {Cüppers, }}$ where $l_{\text {Cüppers }}$ is the distance between the natural insertion point of the muscle and the suture. Except from that modification, all the formulas derived above stay the same.

Figure 4 shows a case where $l_{\text {Cüppers }}>l_{\text {mg }}$. In that case $\Phi_{\max }$ $<0$ (in equation (2)), which means that already for looking straight ahead $(\delta=0)$ the force component generating the torque, $F_{\text {tang, }}$ is smaller than $F$ (equation 4 ).

After the Fadenoperation, we obtain for the force generating the torque, $F_{\text {tang }}$, from equation 4

$$
F_{\text {tang }}=F * \cos \left(\alpha_{\text {cuep }}\right)
$$

where

$$
\alpha_{\text {cuep }}=\delta+\frac{l_{\text {cüppers }}-l_{\text {mg }}}{r_{\text {eye }}}
$$

For an eye movement of the magnitude $\alpha_{\text {cuep }}=90^{\circ}$, the situation reaches a limit (fig $4 \mathrm{~B}$ ): $F_{\text {tang, }}$ which rotates the eye, has been reduced to zero: no rotating torque acts on the eye, and the whole force $F=F_{\text {rad }}$ pulls on the eyeball. Not too surprisingly, this can lead to deformations and retraction of the globe, as well as to the retinal detachments reported in the literature. . $^{-11} 2930$

Pulleys are changing the pulling direction of an EOM. Mathematically, this requires a replacement of $\delta$ by $(\delta-$ $\left.\delta_{\text {pulley }}\right)$ in equation $8 . \delta_{\text {pulley }}$ depends on the exact pulley location, which can be found by magnetic resonance imaging. ${ }^{42}$

\section{Y-split recession}

For a reduction of the torque $T$ it is necessary to change either the force $F$ or the lever arm $r$. The Y-split recession technique is aimed at reducing the torque on the eye by reducing the lever arm. ${ }^{19}{ }^{20}$ This corresponds to the situation in figure 2C, where the seesaw gets balanced by moving the heavy child closer to the centre of the rotation.

This lever arm reduction can be achieved in the following way (fig 5): firstly, the muscle is split with scissors at a length of 15-17 mm. The two halves are then each tied together, and detached from the globe. They are re-inserted at a certain distance from each other, as shown in figure 5A. Surgical procedures and additional diagrams and details are presented in the accompanying article. ${ }^{31}$ A view from above shows that both muscle parts slide to the side, thereby reducing the effective lever arm (fig 5B, broken line). This mechanism is effective over the full oculomotor range (fig 5B). ${ }^{19}$

The muscle and pulley situation after the operation is illustrated in figure 6, in a 17 year old subject. The location of the EOM pulleys remains unchanged, and the operated muscle is able to act with reduced torque.

Mathematically, the effect of a Y-split recession can, to a first approximation, be described by reducing the radius from $r_{\text {eye }}$ to $r_{\text {eff, }}$, as indicated in figure 5C. So without changing the
A Side view

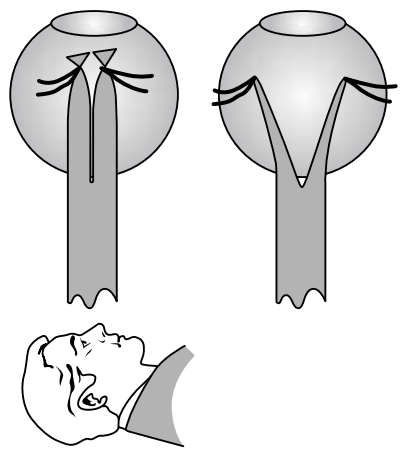

B Top view

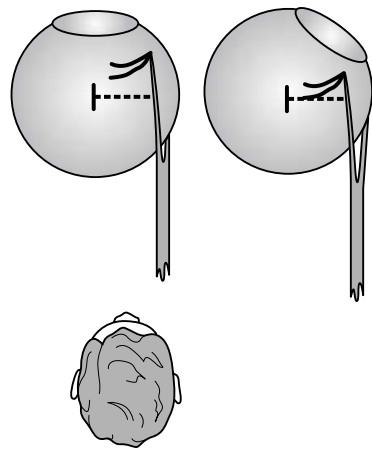

C Lever arm

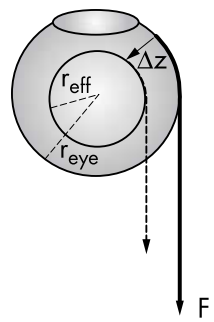

Figure 5 Y-split recession technique. (A) Side view. The muscle is divided at a length of $15 \mathrm{~mm}$ (left), and both muscle parts are reinserted into the globe at a distance from each other (right). (B) Top view: since the muscle parts slide to the side, the lever arm (broken line) is reduced (left). In eccentric eye positions there is no negative effect as with the Cüppers technique (right). (C) Lever arm: $r_{\text {eye }}$ radius of the eye; $r_{\text {eff }}$ effective radius after $Y$-split recession; $F$, muscle force; $\Delta z$, vertical replacement. 

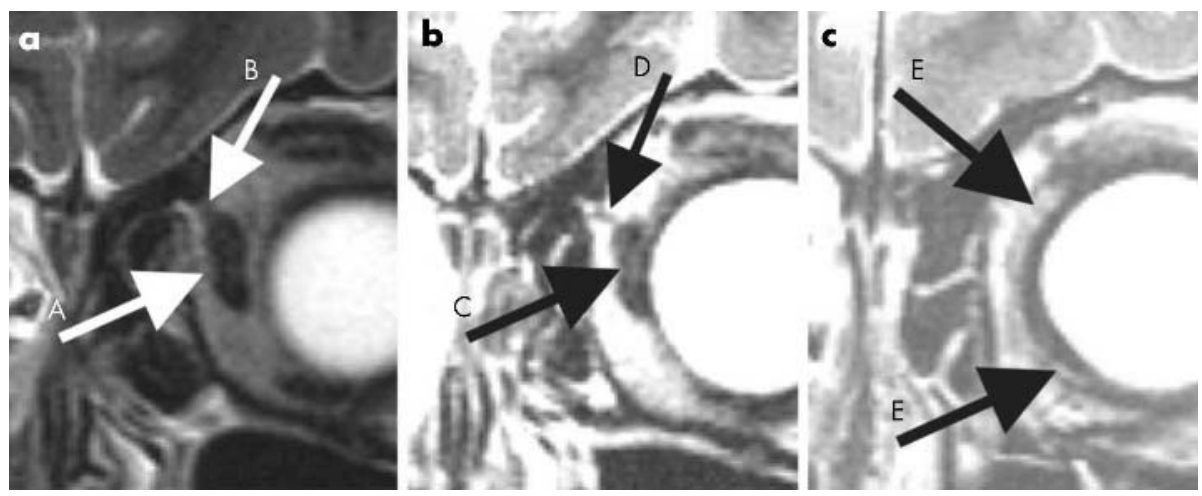

Figure 6 MRI images 9 years after a Y-split recession: (a) muscle (A) is not split in the area of the pulley (B), (b) Muscle split point (C) and ending pulley (D), (c) the two muscle parts (E) immediately posterior the new insertion points. Different MRI data are used for each image for better contrast. Image $b$ and $c$ are located 2 and $14 \mathrm{~mm}$ anterior to image $a$, respectively.

force $F$ of the EOM, the torque exerted on the eye is reduced from $T=F^{*} r_{\text {eye }}$ (equation 3 ) to

$$
T_{\text {eff }}=F * r_{\text {eff }}
$$

For a displacement of the changed muscle by a distance of $\Delta \mathrm{z}$ along the globe (indicated by the broken arrow/smaller circle in fig $5 \mathrm{C}$ ), the effective radius $\mathrm{r}_{\mathrm{eff}}$ becomes

$$
r_{\text {eff }}=r_{\text {eye }} * \cos (\theta)
$$

with

$$
\theta=\Delta z / r_{\text {eye }}
$$

\section{Muscle recession}

For small recessions with a muscle fixation along the arc of contact, the main effect is a concomitant reduction of the force $F$ : by reducing the length of the muscle, the lengthtension relation of the extraocular muscles leads directly to a force reduction. For larger recessions, with muscle fixation behind the arc of contact, the same geometrical concepts apply as for the Cüppers operation. The torques can be calculated in the same way as described above, and for larger eye movements the force reduction becomes incomitant.

\section{RESULTS}

To compare the effects of a Y-split recession with those of a Fadenoperation, we have calculated the torque $\mathrm{T}$ (equation 3 ), as well as the radial force acting on the eye (equation 6) for the two techniques. For a Y-split recession, we used a torque reduction of $25.9 \%$. And for Cüppers' Fadenoperation we chose a situation where the muscle suture was placed $15 \mathrm{~mm}$ behind the natural insertion. The results are presented in figure 7 .

When the subject is looking straight ahead (eye position $=0^{\circ}$ ), the effects of the Cüppers operation and Y-split recession are similar, and the rotating torque is reduced in both cases (fig 7A). But looking at figure 7B, we can see immediately that the two surgeries are not equivalent: while in the case of Y-split recession the radial force pulling on the eyeball is the same as in healthy subjects, a patient experiences a significant radial pull on the eyeball after a Cüppers operation. Mathematically, Cüppers' Fadenoperation with the parameters given above shifts the torque and force curves of a normal eye ( solid line in fig 7 ) to the left, by:

$$
\frac{l_{\text {cueppers }}}{r_{\text {eye }}} * \frac{180}{\pi}=70^{\circ}
$$
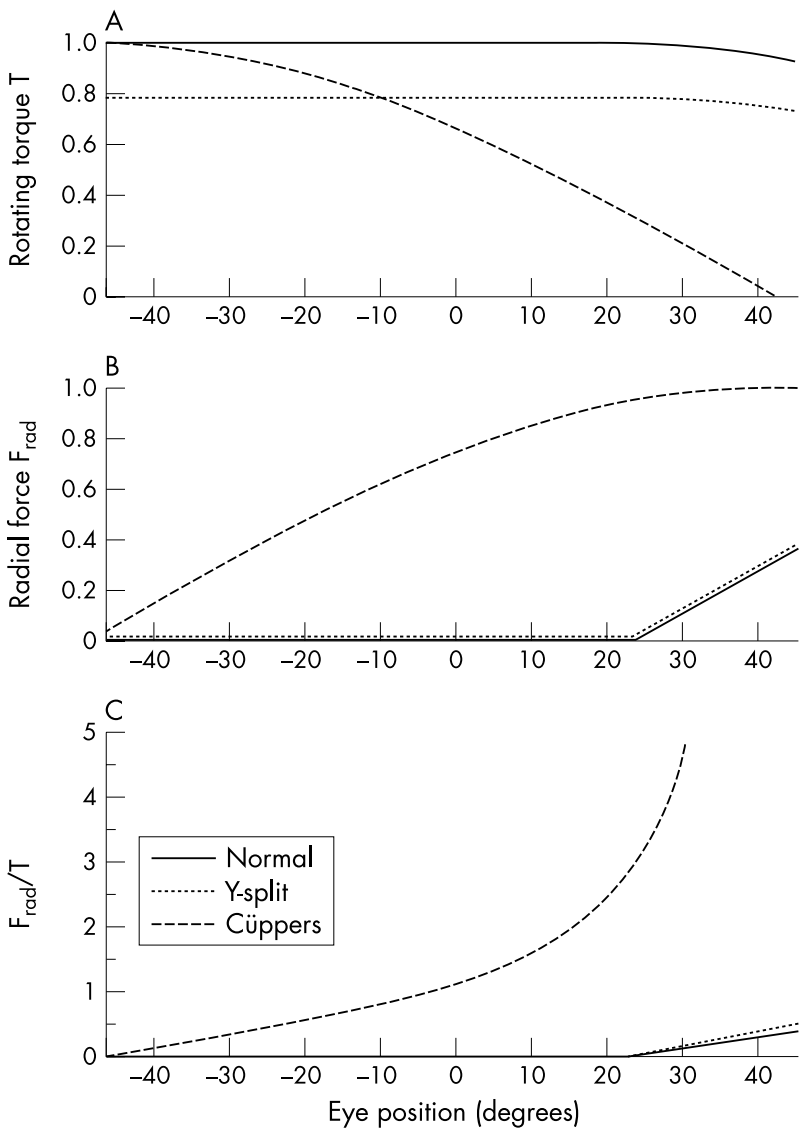

Figure 7 (A) Torque exerted by the extraocular muscle onto the eyeball, for eye positions from $-45^{\circ}$ to $45^{\circ}$. The solid line represents the results for a healthy subject; the dotted line indicates the torque after a $Y$ split recession, and the broken line after a Cüppers' Fadenoperation (parameters given in the text). The torque is normalised to 1 for a healthy subject looking straight ahead. (B) The corresponding radial force $F_{\text {rad. }}$ (C) Ratio between radial force and rotating torque: note the dramatic increase of this ratio after Cüppers' Fadenoperation, for rotations towards the operated muscle. 
A Without...

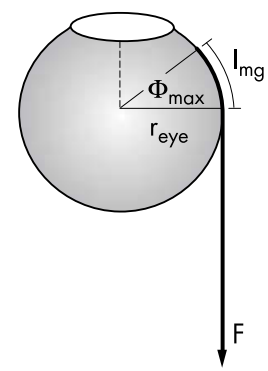

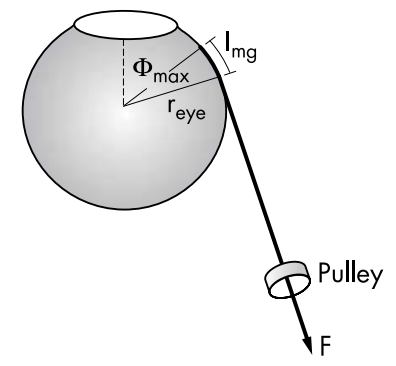

B ...and with pulley

Figure 8 Pulleys. The inclusion of EOM pulleys does not affect the validity of our approach. The main effect is a reduction of $I_{\mathrm{mg}}$.

In contrast, a patient treated with Y-split recession only experiences a reduced torque. ${ }^{19}$

The differences between the two techniques get even more pronounced when we compare the useful rotating torque with the inappropriate radial force (fig 7C). The dramatic difference between Y-split recession and Cüppers, especially for rotations towards the operated muscle, becomes immediately visible. While consideration of pulley locations based on MRI images would lead to a broken line representing the situation after a Cüppers-Fadenoperation in figure 7C that increases somewhat less dramatically, ${ }^{26}$ the large difference between Cüppers- and Y-split recession traces would still be very pronounced.

\section{DISCUSSION \\ Pulleys}

Recent research on the functional anatomy of the extraocular muscles has revealed that the rectus muscles are not homogeneous structures. They can be subdivided into a global and an orbital layer. ${ }^{32}$ While the global layer inserts into the globe, and through contraction exerts the rotating torque on the eye, the orbital layer inserts into the connective structures that attach the EOMs to the orbital wall. ${ }^{33}$ These structures are now generally referred to as "extraocular muscle pulleys," and a recent investigation has demonstrated that they have a pronounced effect on the direction of the forces exerted on the globe after a fadenoperation. ${ }^{34}$ We therefore don't expect that the calculations presented above exactly represent the forces acting on the eye. However, such simplified representations allow us to separate the effect of different surgical and/or medical procedures on the oculomotor system, which in turn helps us to optimise the treatment of patients with strabismus. Approximating muscles as strings has been used successfully by Robinson, ${ }^{35}$ Miller, ${ }^{36}$ and Simonsz, ${ }^{37}$ to investigate the basic mechanical properties of the oculomotor system. To incorporate the detailed geometry and physiology of the oculomotor plant, we collaborate with researchers working on a detailed mechanical model. ${ }^{24} 25$

From a mathematical point of view, pulleys leave the formulas derived above to a first approximation unchanged. A muscle pulley would mainly change the pulling direction of the muscle, which can easily be incorporated by reducing $l_{m g}$ in equation 2 (see fig 8 ); the remaining formulas stay the same. This shifts the curves in figure 7 horizontally, but otherwise leaves them unaffected. The same argument is valid for changes in the pulling direction of a muscle, an effect also addressed by Clark $e a^{38}$ : it would lead to a small horizontal translation of the curves, without changing their shape.

A somewhat more complicated effect is introduced by the proximity of the muscle origin to the eyeball. In our simplified simulations we have assumed that the muscles are infinitely long. For the Fadenoperation, curves that include the effects of a "short" EOM have been presented by Scott, although without giving the exact formulas. ${ }^{39}$ Similarly, considering short splits of the muscle introduces a complicated mechanical behaviour, since for rotations away from the operated side a coiled up muscle would increase the lever arm. Since these effects lead to a significant increase in complexity, but do not introduce any essential change in the underlying mechanics, we do not consider them here. For the same reasons, length-tension relations have not been considered in our simplified model.

\section{Anatomical and physiological advantages of Y-split recession}

With a Y-split recession, the globe area that needs to be accessed is a maximum of $6 \mathrm{~mm}$ behind the natural muscle insertion. Splitting the muscle with scissors can be done without affecting the surrounding tissue. This means that the integrity of the pulley sleeves, which are essential for maintaining the correct pulling directions of the EOMs, are not affected by the operation. Even muscle splits of more than $15-17 \mathrm{~mm}$ can be performed in such a way that splitting would not affect the pulley structures of the medial rectus muscle. The anterior location of the area that has to be accessed during surgery also benefits the operation, which is easier to execute than operations on more posterior locations. In contrast, Cüppers' Fadenoperation typically attaches the EOM $15 \mathrm{~mm}$ behind the muscle insertion, an area that is much less accessible. This increases the risk of sclera perforations. As Clark and Demer have shown, EOM transpositions with posterior fixation can also significantly change the geometry of the oculomotor plant. ${ }^{40}$

The functional advantage of Y-split recession over Cüppers' Fadenoperation is clearly visible in figure 7: while the effect of Cüppers' Fadenoperation depends strongly on the orientation of the eye, Y-split recession achieves a more even effect. As a result some disadvantages of the Fadenoperation can be avoided: the loss of globe motility, caused by the abrupt decrease of rotating torque for rotations towards the operated muscle (fig 7A, broken line), does not show up after Y-split recession operations; and the large radial forces (fig 7B), which are responsible for globe retraction, deformation and detachment of the retina, are effectively reduced by Y-split recession. Y-split recession preserves normal ductions and convergence, in contrast with the Fadenoperation.

\section{Authors' affiliations \\ T Haslwanter, Department of Neurology, University Hospital Zürich, Switzerland and Upper Austrian Research, Medical Informatics, Linz, Austria \\ R Hoerantner, S Priglinger, Krankenhaus der Barmherzigen Brüder Linz, Austria}

Correspondence to: Thomas Haslwanter, PhD, Upper Austrian Research $\mathrm{GmbH}$, Medical-Informatics, Softwarepark Hagenberg, Hauptstrasse 99, A-4232 Hagenberg, Austria; thomas.haslwanter@uar.at

Accepted for publication 9 April 2004

\section{REFERENCES}

1 Dyer JA, Lee DA. Atlas of extraocular muscle surgery. New York: Praeger, 1984.

2 Boschi MC. Nystagmus surgical treatment by means of M Focositendon lenthening. Boll Ocul 1991;70:363-6.

3 De Decker W, S Kaufmann H. Bi-medial rectus muscle resection for intermittent exophoria. Brussels: Transactions 20th Meeting European Strabismological Association, 1992:317.

4 Demer JL, Miller JM, Poukens V. Surgical implications of the rectus extraocular muscle pulleys. J Pediatr Ophthalmol Strabismus 1996;33:208-18. 
5 Cüppers C. Korrektur der Horizontalabweichung. Berufsverband der Augenärzte Deutschlands: Arbeitskreis Schielbehandlung, 1972;5:11-19.

6 Cüppers C. Moderne Schielbehandlung. Klin Monatsbl Augenheilkd 1956;129:579-604.

7 Cüppers C. Grenzen und Möglichkeiten der pleoptischen Therapie. Klin Monatsbl Augenheilkd 1961;381:33.

8 Cüppers C. Some reflections on the possibility of influencing the pathological fixation act. Ann Roy Coll Surg Engl 1966;38:308-25.

9 Cüppers C. The so-called Fadenoperation. Marseille, Kimpton: Second ISA congress, 1974

10 Stein A, Sradj N. Six years of experience with structural dissociated surgery Transactions 19th Meeting European Strabismological Association, 1991:71-5.

11 Thomas Ch. Erfahrungen mit der Fadenoperation nach Cüppers. Arbeitskreis Schielbehandlung Berufsverband der Augenärzte Deutschlands, 1977;9:107-17.

12 Roggenkämper $\mathbf{P}$, Sipp V. Bulblänge und Dosierung der Fadenoperation. Arbeitskreis Schielen Berufsverband der Augenärzte Deutschlands 1980;12:23-6.

13 Clark RA, Isenberg SJ, Rosenbaum AL, et al. Posterior fixation sutures: a revised mechanical explanation for the fadenoperation based on rectus extraocular muscle pulleys. Am J Ophthalmol 1999;128:702-14.

14 De Decker W. The Faden operation. When and how to do it. Trans Ophthalmol Soc UK 1981;101(P+ 2):264-70.

15 Leitch RJ, Burke JP, Strachan IM. Convergence excess esotropia treated surgically with fadenoperation and medical rectus muscle recessions. Br J Ophthalmol 1990;74:278-9.

16 Carruthers JD, Kennedy RA, Bagaric D. Botulinum vs adjustable suture surgery in the treatment of horizontal misalignment in adult patients lacking fusion. Arch Ophthalmol 1990;108:1432-5.

17 Keech RV, Morris RJ, Ruben JB, et al. Anterior segment ischemia following vertical muscle transposition and botulinum toxin injection. Arch Ophthalmol 1990;108:176.

18 Leigh RJ, Tomsak RL, Grant MP, et al. Effectiveness of botulinum toxin administered to abolish acquired nystagmus. Ann Neurol 1992;32:633-42.

19 Priglinger S, Hametner H. Operative Methoden zur Behandlung des schwankenden Schielwinkels. Mathematische ZusammenhängeComputergestützte Operatioinsplanung. Spektrum Augenheilkd 1994;8:162-75.

20 Günther S. Die modellmssige Beschreibung der Augenmuskelwirkung. 1986 University Hamburg, Fachbereich Physik, Augenklinik.

21 Clark RA, Isenberg SJ, Rosenbaum AL, et al. Posterior fixation sutures: a revised mechanical explanation for the fadenoperation based on rectus extraocular muscle pulleys. Am J Ophthalmol 1999;128:702-14.

22 Demer JL, Oh SY, Poukens V. Evidence for active control of rectus extraocular muscle pulleys. Invest Ophthalmol Vis Sci 2000;41:1280-90.
23 Miller JM, Shamaeva I, Pavlowski DS. Orbit 1.8 gaze mechanics simulation. San Francisco: Eidactics, 1999.

24 Buchberger M, Mayr H. SEE-KID: software engineering environment for knowledge-based interactive eye motility diagnostics. Proceedings of the International Symposium of Telemedicine, 2000.

25 Buchberger M. Ein biomechanisches Modell der Augenmotilität. Spektrum Augenheilkd 2002;16:176-82.

26 Clark RA, Isenberg SJ, Rosenbaum AL, et al. Posterior fixation sutures: a revised mechanical explanation for the fadenoperation based on rectus extraocular muscle pulleys. Am J Ophthalmol 1999;128:702-14.

27 Clark RA, Isenberg SJ, Rosenbaum AL, et al. Posterior fixation sutures: a revised mechanical explanation for the fadenoperation based on rectus extraocular muscle pulleys. Am J Ophthalmol 1999;128:702-14.

28 Leitch RJ, Burke JP, Strachan IM. Convergence excess esotropia treated surgically with fadenoperation and medical rectus muscle recessions. Br J Ophthalmol 1990;74:278-9.

29 Alio JL, Faci A. Fundus changes following faden operation. Arch Ophthalmol 1984;102:211-3.

30 Bialasiewicz AA, Ruprecht KW, Naumann GO. Staphylococcal endophthalmitis following squint surgery. Klin Monatsbl Augenheilkd 1990;196:86-8.

31 Hoerantner R, Priglinger S, Haslwanter T. Reduction of ocular muscle torque by splitting of the rectus muscle I: Technique and results. $\mathrm{Br} J$ Ophthalmol 2000;88:1409-13.

32 Oh SY, Poukens V, Demer JL. Quantitative analysis of rectus extraocular muscle layers in monkey and humans. Invest Ophthalmol Vis Sci 2001;42:10-16.

33 Kono R, Clark RA, Demer JL. Active pulleys: magnetic resonance imaging of rectus muscle paths in tertiary gazes. Invest Ophthalmol Vis Sci 2002;43:2179-88

34 Clark RA, Isenberg SJ, Rosenbaum AL, et al. Posterior fixation sutures: a revised mechanical explanation for the fadenoperation based on rectus extraocular muscle pulleys. Am J Ophthalmol 1999;128:702-14.

35 Robinson DA. A quantitative analysis of extraocular muscle cooperation and squint. Invest Ophthalmol 1975;14:801-25.

36 Miller JM, Robinson DA. A model of the mechanics of binocular alignment Computers and Biomedical Res 1984;17:436-70.

37 Simonsz HJ. Robinson's computerized strabismus model comes of age. Strabismus 1996;4:25-40.

38 Clark RA, Isenberg SJ, Rosenbaum AL, et al. Posterior fixation sutures: a revised mechanical explanation for the fadenoperation based on rectus extraocular muscle pulleys. Am J Ophthalmol 1999;128:702-14.

39 Scott AB. The faden operation: mechanical effects. Am Orthopt J 1977;27:44-7.

40 Clark RA, Demer JL. Rectus extraocular muscle pulley displacement after surgical transposition and posterior fixation for treatment of paralytic strabismus. Am J Ophthalmol 2002;133:119-28. 